NUMERALISED PROFILES FOR CLASSIFICATION AND RECOGNITION.

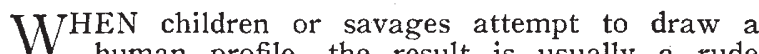
human profile, the result is usually a rude fgrure that lays stress on five cardinal points. These are the notch between the brow and the nose, the tip of the nose, the notch between the nose and the upper lip, the parting of the lips, and the tip of the chin. Supposing these five points, $B^{\prime}, N^{\prime}, U^{\prime}, L^{\prime}$, and $\mathrm{C}^{\prime}$, to be located with fair precision, as will shortly be shown to be feasible, then Fig. I is directly deducible from them, together with the vertical and horizontal axes, $C^{\prime} B^{\prime}$, and $C^{\prime} X$ at right angles to $C^{\prime} B^{\prime}$. The position of the five cardinal points varies in different profiles much more than the probable error of measurement. So though Fig. I is a mere skeleton, which determines what may be called the set of the features, and corresponds to the primary triangulation of a country, other points are to be derived from it, and similarly utilised. Among these are the intersections with the outline by perpendiculars, drawn from the middle or other specified division of the lines. This skeleton serves as an excellent basis for the classification of profiles and for anthropological statistics.

Peculiarities of profile, as a racial or family characteristic, can be expressed numerically by an extension of this system in a way that promises to be serviceable for eugenic records. It was, in fact, largely with this object in view that I began the

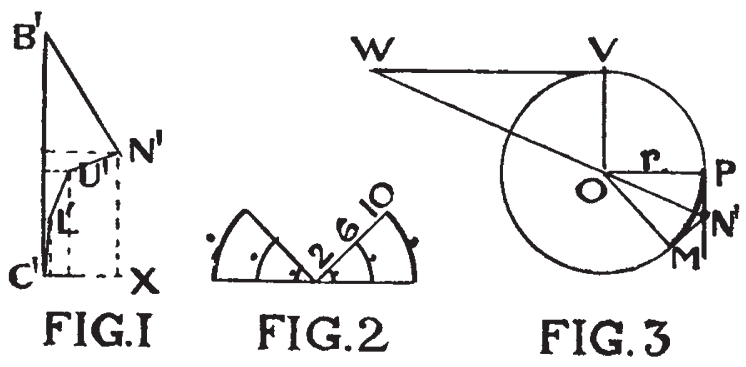

inquiry. The replacement in all scientific work by numerical values, in the place of vague adjectives, is a gain of first-class importance. There is no way known to me, other than this, by which likenesses can be "lexiconised," that is, arranged as words in a dictionary. A needed portrait may by its means be discovered by a formula, as a spoken word is found in a dictionary, by the letters that express its sound. There are many simple purposes of newspaper interest to which this same method might be applied, but with more elaboration.

The practice of cataloguing profiles may perhaps become useful as a secondary means of identification when the number of persons who may require to be identified shall have become too large to be readily dealt with by finget-prints alone.

It will be shown (Fig. 5) that four telegraphic "words" are sufficient to convey a very fair profile likeness. The cost of sending an extra four words by telegram to any part of the British Isles being only twopence, and of a moderate amount over-seas, the practice of telegraphing profiles of persons of current interest, might become common. A refugee criminal could easily be outstripped by his portrait, sufficiently like to him to justify, in connection with corroborative evidence, his being placed for a while under police observation. The measures of profiles must, of course, be reduced to uniformity. Thus, by utilising two out of the five cardinal points to give direction and scale, the mean positions of the remaining three No. 2 I09, VOL. 83] points may be determined for any given race or family, together with the frequency of deviations of any given amount from those mean positions, and such other deductions as can.be reached by the modern methods of statistics.

The corrected values are here described by the same letters as the original ones, but without the dashes. The standard scale that is used is such that $\mathrm{BC}$, the corrected value of $\mathrm{B}^{\prime} \mathrm{C}^{\prime}$, shall be always 50 units in length (see Fig. 5). The reduction is, of course, effected by multiplying each measure in the portrait by 50 divided by its $\mathrm{B}^{\prime} \mathrm{C}^{\prime}$. The number 50 is preferable to IOO, which would probably first suggest itself, for a variety of practical reasons, into which I need not now enter. Two figures are assigned to each measure, so the values $0, \mathrm{I}, 2,-.9$, have to be written oo, oI, 02, .09. The measures are recorded to the nearest integer, there being no room for fractions, decimal or other. A millimetre is a convenient unit for purposes of drawing, more so than one-tenth of an inch; therefore, in reproducing the corrected measures, BC becomes 50 millimetres, and the other measures are altered in the same proportion.

A thick beard interferes with determining $L^{\prime}$ and $\mathrm{U}^{\prime}$, but their positions can usually be inferred with a useful degree of precision in moderately bearded faces.

The accuracy with which the five cardinal points can be located differs considerably. The most exact determinations in an unbearded face are those of the points $\mathrm{C}^{\prime}$ and $\mathrm{N}^{\prime}$, and the direction of the line $\mathrm{C}^{\prime} \mathrm{B}^{\prime}$. $\mathrm{U}^{\prime}$ comes next in order of exactness, then $\mathrm{B}^{\prime}$, and, lastly, $L^{\prime}$. The distance between a line joining $\mathrm{C}^{\prime} \mathrm{N}^{\prime}$ and a parallel line tangential to $\mathrm{U}^{\prime}$, can be fixed with precision but is not used here. $\mathrm{C}^{\prime}$ and $\mathrm{N}^{\prime}$ are each defined by the intersection of two tangents, as shown for $\mathrm{N}^{\prime}$ in Fig. 3 .

It is well to examine these conditions more closely, as they bear on the treatment of curvatures generally. A knowledge of them permits rough and ready drawing, in which the principal matters are attended to, the less essential ones being more or less disregarded. One of the tangents is parallel to $C^{\prime} B^{\prime}$, which is treated as vertical; the other is inclined to the vertical at $45^{\circ}$. Consequently, the curve of $\mathrm{N}^{\prime}$ is contained in an obtuse angle of $180^{\circ}-45^{\circ}=135^{\circ}$. The tip of each prominence and the bottom of each hollow is represented by one or other of the three short circular arcs shown in Fig. 2, which are sufficiently numerous for the purposes to which they are here applied. The centres of all circles that touch both the vertical and the diagonal will necessarily lie in the line that bisects the obtuse angle between them; consequently, $N^{\prime} O$ forms an angle with the vertical that is equal to half $135^{\circ}$, or $67 \frac{1}{2}^{\circ}$. The tangent of this angle is 2.4142 ; therefore the position of the line of centres may be found by laying off a point $V$ in a vertical direction, at Io units of length from $O$, and by drawing another line from $\mathrm{V}$ horizontally to $\mathrm{W}$, at a distance from it equal to 24. I4 of the same units as before. Then the line of centres passes through $O$ and W. It is easily shown (Fig. 3) that the points of contact between the circle and the two tangents are exactly $45^{\circ}$ of arc apart. The length of the chord of that angle is equal to about three-quarters of its radius. The shortness of the chord, when the radius is small, is well seen in Fig. 2, and must be borne in mind; it accounts for the scarcely noticeable differences in the curvatures, and consequently for the fewness of the standard arcs that are necessary. The arc of $45^{\circ}$ is shown by a heavy line in Fig. 3 , where the circle has a radius of ro $\mathrm{mm}$. There is often a colloquial confusion between the obliquity of the planes between which an edge lies and that of the sides of the edge itself. The former may be very acute, and the angle of the edge would be equally acute if the planes were prolonged until they met; but usually they do not meet, the edge itself being more or less rounded. The acutely inclined faces of a knife may have a blunted edge, that fails to cut the skin without much pressure, while a broken piece of glass, the fracture 
of which is perpendicular to its face, but the edge of which is not blunted, only too readily makes a gash.

The arcs of $45^{\circ}$ in Fig. 2 all refer to the cutting edge, so to speak. The direction of the lines within which the cutting edge is situated is determined by the adjacent cardinal points towards which they point.

Referring back to Fig. 3, $O P=O M=r$, the radius, $\mathrm{ON}=r \times \mathrm{r} .082$, therefore the distance between $\mathrm{N}$ and the circle is only $r \times 0.82$, which for a radius $=10 \mathrm{~mm}$. is about three-quarters of I $\mathrm{mm}$. This small value is diminished in proportion for lesser values of $r$ (see Fig. 2), so for practical purposes $\mathrm{N}$ (and similarly $\mathrm{C}$ ) may be considered to lie only just outside the convex circumference of the arc by which they are in each case represented.

The values of $r$ which are used as standards for the lesser curvatures are $2 \mathrm{~mm}$., $6 \mathrm{~mm}$., and Io $\mathrm{mm}$. The drawings in Fig. 4 are not exactly on this scale, but the differences are unimportant. It is unnecessary to divide these small curves into concave and convex, as their condition in that respect is indicated by the part they play in the profile. Two other curvatures of the larger radii, $25 \mathrm{~mm}$. and $30 \mathrm{~mm}$., are used to express and to define the concavity or the convexity of the ridge of the nose.

It is well, at the risk of some repetition, to describe in a single paragraph the nomenclature of the five cardinal points in the original portrait. $\mathrm{B}^{\prime}$ is the point in the fronto-nasal notch at what is judged to be its deepest part; $\mathrm{N}^{\prime}$ is the tip of the nose, found in the way already described; $\mathbf{U}^{\prime}$ is the point of contact between the naso-labial notch and a tangent, drawn diagonally to it; $L^{\prime}$ is a point half-way between the furthest positions at which the lips would touch one another if they were lightly closed; $\mathrm{C}^{\prime}$ is found by a similar method to that used for $\mathrm{N}^{\prime}$.

The portraits are described by numerical formulæ. Each formula consists of four groups of figures, five figures in each group. The shapes of the profile at and immediately adjacent to the cardinal points, and those of the intermediate links, are expressed by single numerals, as set forth in tabular form in Fig. 4. Not more than o to 9 , or 10, varieties of shape are provided in each case. Thus, the radius of the standard curve that best fits the frontonasal notch, $b$, is expressed by its appropriate numeral, as shown in the first line of Fig. 4; also the inclination of the brow immediately above $b$, whether it slopes forwards, backwards, or is upright. The ridge of the nose $g$ is counted as either sinuous, concave, or convex, in two or three different degrees, or else as straight. The letter $n$ includes both the very tip of the nose and the outline underneath it, which leads towards the naso-labial notch. The letter $u$ includes the nasolabial notch and the first portion of the upper lip. The lips require two statements, and therefore two separate figures; the former, $l p$, shows whether the lips are shut, parted in the portrait by I or $2 \mathrm{~mm}$., or open by $3 \mathrm{~mm}$. or more, and, again, whether they project evenly, are overhung or underhung. The latter notation, $l l$, expresses the sizes of the upper and lower lips respectively, whether they are small, medium, or large. The outline between the lower lip and the chin is always notched, and $k$ describes the size and Fosition of the notch, whether it be small, medium, or large, and whether it be high, medium, or low. 'The curve of the chin itself at $\mathrm{C}^{\prime}$ is not given.

I have called these profiles "numeralised" to express the fact that they are transformed into numerical NO. 2109 , VOL. 83 ] formulæ. Twenty figures enter into each formula; they are arranged, as for telegraphy, in the way already described, into four groups of five figures in each group. A "figure," in telegraphic language, includes not only the ten numerals, o to 9 , but the three symbols in addition, of a stop (.), a hyphen $(-)$, and a short oblique line (/), such as is used in fractions. The arrangement in groups of five, or in "quintets," proved suitable to other similar work on which I was engaged, so it has been adopted throughout. In the four quintets, or, we may say, in the four words that compose a formula, the first three refer respectively to $\mathrm{N}, \mathrm{U}$ and $\mathrm{L}$, and in that order. The first two and the last two figures, in each of the first three quintets, give the position of the point in question in $\mathrm{X}$ and in $\mathrm{Y}$ to the nearest whole millimetre. The middle figure of the quintet is derived from Fig. 4 to describe the peculiarities of the profile at and immediately adjacent to that point. The fourth and last of the quintets is preceded by a dot (.), to show that it belongs to a separate category, $\begin{array}{llllllllll}2 & 3 & 4 & 5 & 6 & 7 & 8 & 9 & 0\end{array}$

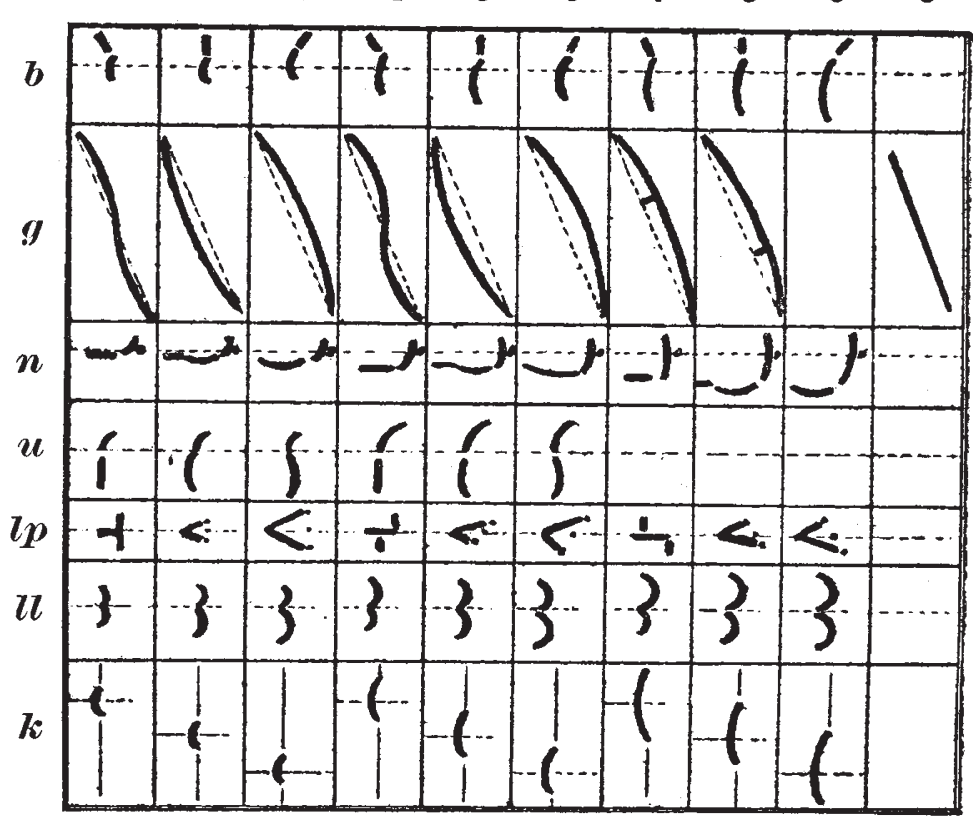

FIG. 4.

namely, to the peculiarities of $b, g, l l$, and $k$, as set forth in Fig. 4, and in that order. When proceeding to draw a figure from a formula, it is advisable for a beginner to use tracing paper. Then, after drawing an arbitrary line in any convenient place, of 50 millimetres in length, to serve for $\mathrm{BC}$ and, therefore, for the vertical axis, and another line $\mathrm{CX}$, at right angles to $\mathrm{CB}$ at $\mathrm{C}$, for the horizontal axis, to plot the positions of $\mathrm{N}, \mathrm{U}$, and $\mathrm{L}$; then, laying the transparent paper upon Fig. 4, to trace, or copy on an enlarged or reduced form, according to the space available, the figures of $n, u$, and $l p$, very faintly. Next to do the same to $b, g, l l$, and $k$. Afterwards to harmonise the whole tentatively, with faint and brush-like strokes; lastly, with a free and firm hand to draw the outline through them. Tracing paper may otherwise be convenient, because when the original profile looks to the left, by the simple act of turning the traced outline it affords an almost equally clear profile, looking to the right. 
When transforming the portrait into a formula, the reverse process has to be followed with little alteration. Before finally adopting any formula, the portrait should be reconstructed from it and the formula revised where necessary. It is easy after a little practice mentally to compose a formula so far as the seven small letters are concerned, from a brief inspection, either of the picture or of the living face; also to reproduce by copying by eye the symbols from Fig. 4 without caring to trace them. In short, the whole operation may be satisfactorily gone through by an traits are by no means deficient in resemblance to their originals. I think they are considerably more like to them than the sketches, usually printed in the illustrated newspapers, are to the public characters whom they profess to represent. They are, to say the least, of considerable negative value, sufficing to eliminate at the rate of about nineteen out of every twenty individuals as not being the pcrson referred to. Any form of telegraphy suffices to transmit these four-word profiles. In ather respects they are far inferior to those complete pictures now transmitted
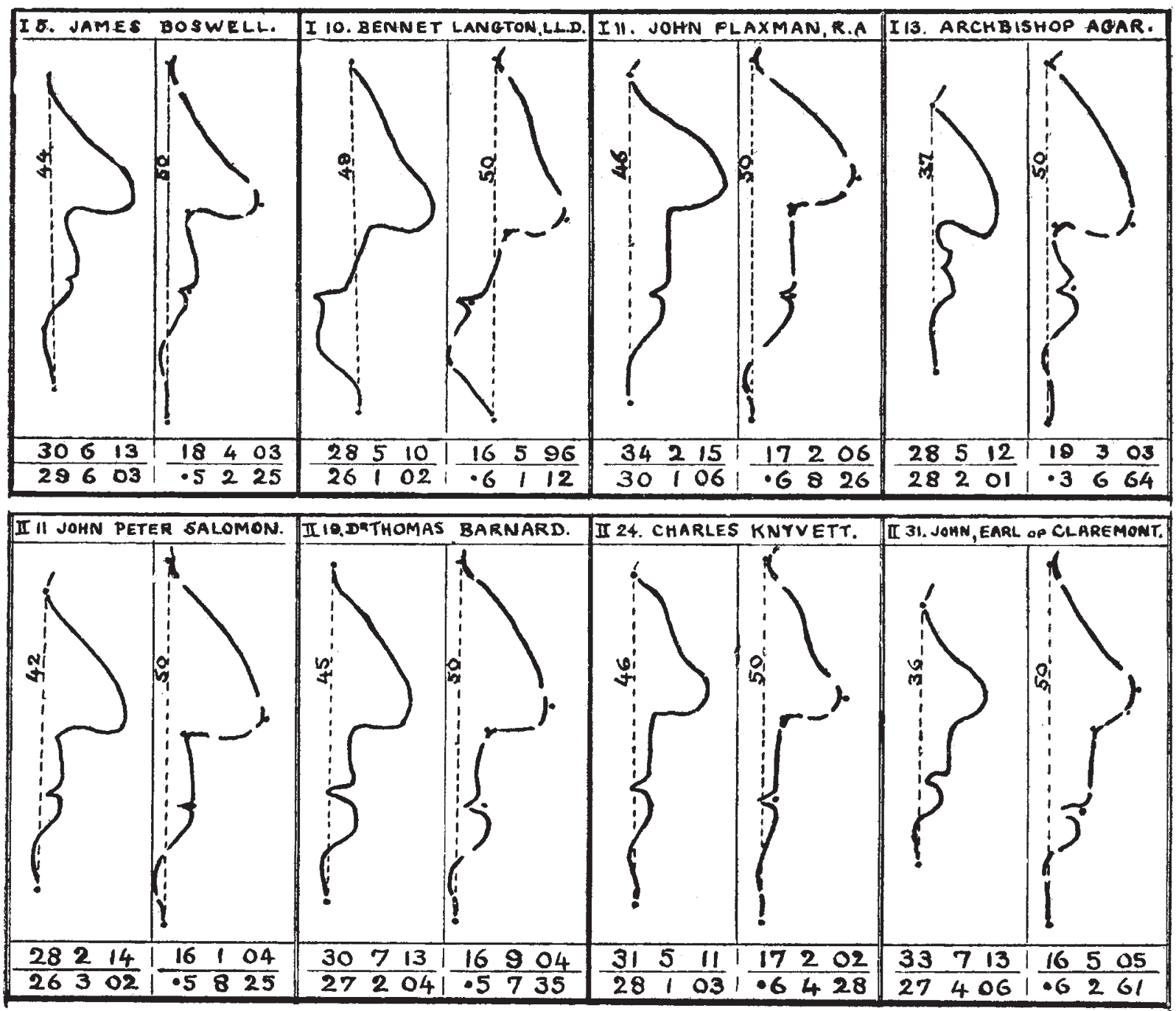

Fig. 5 .

Explanation of the first formula, namely, that of James Boswell; the others are to be read on the same principle. $\mathbf{N}_{x}, 30$; $\mathbf{N}_{y}, 13 . \mathrm{U}_{x}, 28 ; \mathrm{U}_{y}, 03 . \mathrm{L}_{x}, \mathrm{I} 8 ; \mathrm{L}_{y}, 03$. The small letters are, $n, 6 ; u, 6 ; l p, 4 . b, 5 ; g, 2 ; l l, 2 ; k, 5$.

intelligent person in a rapid and off-hand way. This might become a popular game for the members of a party to practise their art upon one another, care being taken that the five cardinal points should be truly laid down, perhaps by tracing a shadow.

Eight couplets of very different features are given in Fig. 5, both for illustration and for the reader to practise upon. Each couplet contains the original portrait on the left, its formula along the bottom, and the reproduction from the formula (to the standard scale) on the right.

It will be seen from Fig. 5 that four-word por- between certain offices, by means of costly and delicate apparatus, by a method at present not developed to its utmost.

It will be observed that in the second of the portraits, namely, that of Dr. Bennet Langton, the point L lies to the left of CY, and has therefore a negative value. This is -04, but is expressed here as 96 , an artifice which practically transfers the horizontal measurement from CY to another vertical line drawn parallel to $C Y$ and $100 \mathrm{~mm}$. to its left. No confusion need arise through this transformation, since it leads to very large values lying adjacent to
No. 2 IOg, VOL. 83]

(c) 1910 Nature Publishing Group 
very small ones, and therefore showing that they belong to a different category. The minus values in $\mathrm{Y}$ are similarly treated. The process may also be extended beyond the eight squares of o to Ioo $\mathrm{mm}$. in their sides, that surround the primary one.

My experiments have been chiefly made upon the "Collection of Portraits by George Dance, R.A., Sketched from Life and Engraved in Imitation of the Original Drawings " (Longmans and Co., I809). They were convenient to work with, being all drawn on scales differing little from that of the standard. All the portraits are unbearded and in exact profile, with three or four exceptions. Those that are available are sixty-eight in number. The name of the person to whom each of the eight portraits in Fig. 5 applies is written along its top, and the volume and page of the two folios by Dance, from which the original was traced, are given in the upper left-hand corners. There are several notabilities in his collection besides those in Fig. 5. Among them are Horace Walpole, General Paoli, Haydn, and John Philip Kemble. An exhibition of Dance's pictures was recently held in London. He had a considerable reputation in his time as a portrait painter.

Methods have been used to aid the recollection of dates and other figures. That by Gray, in his "Memoria Technica," was to transform each numeral into either a consonant or into a vowel or diphthong, as might be the most convenient, and thereby to build up words easy to pronounce and to remember. Those who are familiar with such a process might apply it here, and convert the four quintets of numerals into four words, getting over the difficulty of employing the three additional symbols as best they can. If they succeed, the phrase of "four-word profiles" would be literally exact.

I do not find that a general resemblance can be much increased by using one or a few more quintets or words. A fifth, or even a sixth, quintet might, however, be usefully employed in extending the range of the profile, if it contained one figure to describe the chin and just below it, another to describe the brow, and two figures, oo to 99 , which would perhaps suffice to give the size and general shape of the head, also to define the mustachio and beard of unshaven faces.

The next distinct stage in order of accuracy is separated by a great distance from the present one. It requires so large a number of dots that straight or slightly curved lines drawn through them will flow smoothly when seen at the ordinary reading distance from the eye. It needs as many as perhaps fifty quintets to describe a profile with exactness and the rest of the head with rough precision, and still more to include the eye and ear. I have made many of these, which, when reduced to the standard scale of $B C=50 \mathrm{~mm}$., are practically identical with the originals, when viewed in a somewhat careless way by a normally sighted person at a distance of 12 inches. A special use is made in this case of the middle figure of the quintet. Thus, the numeral I means that a half-unit is to be added to the first two figures; 2 , that it is to be added to the last two; and 3 , that it is to be added to both of them. This power of doubly minute description is often wanted in the outline that joins and includes the nose-tip and the two lips. Another use for the middle figure of the quintet is to tell that a dotted line should be drawn from the preceding point, to signify doubt of some kind. A hyphen (-) in the middle of the quintet means to begin; an oblique line $(/)$ to end; and a point (.) means an isolated point. But I will not go further into this now; neither will I do more than hint at the way of dealing with portraits that are not in exact profile, by multiplying their horizontal NO. 2 IO9, VOL. 83] measures into the secant of the angle through which the profiles are turned away from it.

Much more might be added on extensions of this method, especially as regards its facilities and limitations in conveying plans-ceremonial, strategic, and others-for newspaper use. But its general principles have been explained, and as this article is already too long I will end it abruptly here.

Francis Galton.

\section{TIDAL OBSERVATIONS IN THE ENGLISH CHANNEL AND NORTH SEA.}

FOR the purpose of tracing correctly the progress of the tidal wave throughout its course in the English Channel and North Sea, observations of the vertical movement of the tide at a distance from the land, and similar observations by means of tide gauges on the shore, are equally necessary.

Information on the rise and fall of tide far from the shore may be obtained from a ship or boat at anchor, and in certain localities it may be of considerable value to the navigator.

But, if the observations are carried out with sufficient exactitude to satisfy scientific requirements, the procedure hitherto followed for that purpose necessitates weather conditions which do not often occur, and seldom last long enough for the object in view.

There are other difficulties also to contend with, due to the stream of tide running at its maximum rate at, or about, the times of high and low water. The stream, reversing its direction between those times, causes a mark buoy, or boat, however, tautly moored, to swing over a certain area during the interval; the undulating character of the surface of the ground, and the action of the strong tidal stream on the lead-line, thus tend to introduce elements of uncertainty whicls increase with the depth of water.

The practical difficulties experienced in obtaining strictly accurate results by this means involve loss of time disproportionate to the value of the observations, and therefore the attempt has not often been made.

Trustworthy evidence on the rise and fall of tide is thus almost entirely confined to the coast-line, with the exception of a few observations of tide-gauges attached to the masts of wrecks on off-lying banks in the North Sea.

A large number of observations of value for the reduction of soundings have, however, been obtained in the North Sea during the years $1886-90$ by Captain T. H. Tizard, R.N., C.B., F.R.S., while commanding H.M.S. Triton in the course of the survey of the shoals fronting the Norfolk coast. These, being taken by the lead-line from the ship at anchor, on the assumption that the bottom was perfectly level, can scarcely be considered sufficiently trustworthy for scientific purposes until confirmed by more precise methods which were not then available.

More rigorous observations were carried out in the North Sea by the late Captain W. Hewett, R.N., commanding H.M. surveying ship Fairy in 1838 and 1840 with the object of verifying the prediction of the late Dr. Whewell as to the existence of an area situated eastward of Orfordness and about midway between the coasts of England and Holland, where the rise and fall of tide was expected to vanish.

The method employed by Captain Hewett was to moor a boat head and stern as tautly as possible by means of lead-lines attached to anchors laid out in the direction of the tidal streams. A remarkable elevation in the form of a ridge on the bottom, with a depth of $18 \frac{1}{2}$ fathoms over it, having been previously detected, the boat was moored at slack water as nearly as possible directly over the ridge. Another boat dropping down with the tide, with lead kept just on and 